2018-06-01

\title{
Experiencing Quantum Through Music
}

\author{
Kirke, Alexis
}

http://hdl.handle.net/10026.1/12446

10.1093/itnow/bwy055

ITNOW

Oxford University Press (OUP)

All content in PEARL is protected by copyright law. Author manuscripts are made available in accordance with publisher policies. Please cite only the published version using the details provided on the item record or document. In the absence of an open licence (e.g. Creative Commons), permissions for further reuse of content should be sought from the publisher or author. 


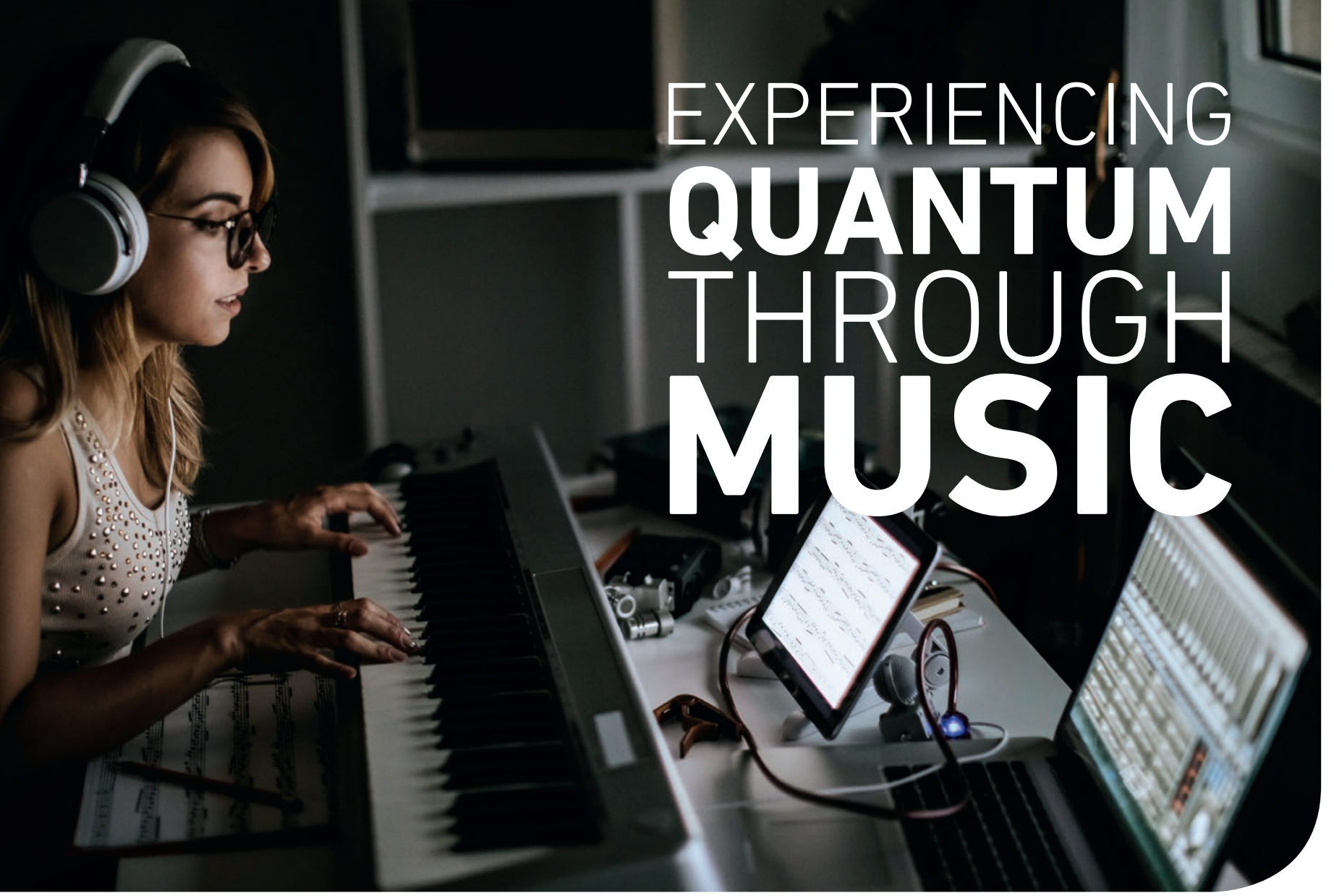

Alexis Kirke, Senior Research Fellow in Computer Music at the University of Plymouth, discusses the general lack of understanding about quantum computers, and an artistic way of introducing more people to it.

News and videos about quantum

computers (QC) are common. 'Quantum' inspires awe and mystery. Astonishing speed-ups are promised. 'Entanglement' is thrown in the mix - people become hooked. But this computer research that inspires such fascination is an area that offers the fewest opportunities for involvement or understanding. Want to learn to programme? Use tools like Scratch. Want to develop machine learning skills? There's a Python package for that. Want to learn about QC? Zip through these courses on complex vector spaces, number theory, and an undergraduate introduction to quantum mechanics. Then you can start trying to understand the basics of QC!

But what about the only 'killer app' for QC - Shor's Algorithm? Well, that would strain the brain of a third-year maths undergraduate. The mysteries of quantum effects are easy to understand in the maths. In the equations all is clear. But it is a mix of maths topics unusual to find in the average computer programmer.

\section{Musical problems}

Another approach to understanding QC involves helping other people understand it. One way to do this is to create musical problems and use QC to solve them.
Discussing the solution to these problems can provide a greater insight into QC.

The example in this article is the musical problem of chords, solved on a quantum D-Wave $2 X$.

The first company to sell quantum computers was D-Wave, who flogged a few off to people such as Google, NASA and Lockheed Martin. The D-Wave computers are adiabatic quantum computers (ADC). They are not like normal algorithmic step-by-step QC, such as those made by IBM. An adiabatic quantum computer is reminiscent of a neural network. It is

came out in areas as wide as zoology and artificial intelligence on the applications of the Ising.

There is an ongoing debate about how the D-Wave ADC truly functions and what speedup it can provide. Google claimed large speed increases for its quantum hardware. This is thought to be due to quantum tunnelling. When searching for low energy states, a quantum system can tunnel into nearby states.

Quantum tunnelling allows physical systems to move to states in ways that would not be possible in the classical

\section{The mysteries of quantum effects are easy to} understand in the maths. In the equations all is clear. But it is a mix of maths topics unusual to find in the average computer programmer.

based on the equations for Ising models. Ising models describe the physics of a magnetic material through the molecules within it.

An ADC solves the equations of the Ising model to minimise the energy in the simulated magnetic material. The programming involves defining properties of the simulated 'molecules'. Over a period of 28 years, more than 10,000 publications
Newtonian view of the world. The systems 'tunnel' through to the new, usually inaccessible states instantaneously.

This particular musical problem was set up by assigning each note of the musical scale to one 'molecule' of the Ising model. Each molecule is modelled by a quantum bit, or Qubit. At this point, the mathematical world of quantum mechanics is entered, where everything makes sense in the 
equations, but not in the explanation!

Every qubit can be simultaneously a one or zero (unlike a bit which can only be one or zero). This is very simple mathematically, but makes no sense in our everyday observed world.

For example, a cat cannot be both alive and dead, as Schrodinger once observed in his famous thought experiment. He was trying to imagine the laws of quantum mechanics applying to the world beyond subatomic particles.

This, so called, 'superposition' of one and zero is not a form of statistical or probabilistic computing. It is something more complex. In the combination of one and zero held by this single qubit, the one and the zero also have what is known as a 'phase'.

This can be thought of as the result of

\section{Qubits in harmony}

The D-Wave $2 X$ dealt with many underlying complexities. Connections were set up between the 'molecules' (the musical notes) in such a way that when the D-Wave program was triggered, it generated the form of musical chord required.

A simple musical rule is used. The D-Wave would be sent a note, and it would find three or four notes which included this note, and which were not too close together nor far apart on the piano keyboard. Try pressing three notes at the same time on the piano keyboard. If they are too close they clash, if they are too far apart they don't sound like a chord.

Each time the D-Wave was asked to harmonise a note using this algorithm, it would send me multiple possible solutions. This highlights a key element of

\section{This, so called, 'superposition' of one and zero is not a form of statistical or probabilistic computing. It is something more complex.}

another strange consequence of quantum theory: everything is simultaneously a wave and a particle. An electron is a waveform, and a light wave is also a particle of light called a photon.

When the qubit is actually measured, its resulting value will always be 0 or 1 . For definite. What's more, the phase of the 0 and 1 in the superposition has no effect on the chance of whether a 0 or 1 is seen. But, until that observation, not only is the result indeterminate, but these phases have dramatic effects on how qubits interact.

Things have clearly moved beyond the realms of how programming is normally thought about. The qubit being like a bit that is both 0 and 1 is a useful analogy, but it's incomplete.
$\mathrm{QC}$ - there is no single correct solution to an algorithm. The solutions are held in a superposition, and then when observed, a single solution presents itself. This is not necessarily the precise process the D-Wave is following, but its qubits move through a number of superpositions as a solution form.

These ideas were captured and explained in a performance at the Port Eliot Music Festival in July 2017 called 'Superposition'. It was a composition for mezzo soprano (Juliette Pochin) and electronic sounds. The electronics were generated by a real-time music system on my laptop, connected over the internet to the D-Wave $2 X$ at USC. The mezzosoprano's music was pre-composed. The sounds of her voice were picked up live by the laptop, converted into energy and frequency readings, and sent to the D-Wave as a problem to be solved by the harmony generator.

The D-Wave returned multiple solutions. The local laptop took the multiple chords, spread them across the musical range, and played them together. These giant chords gave the audience some sense of the multiple solutions that may have existed in the superposition inside the quantum computer.

\section{Universal quantum computers}

The next performance planned will involve the Universal QC (UQC) of IBM. UQC have logic gate diagrams and assembly code. They have processing elements, like NOT, XOR and a form of AND gate.

But... the analogy breaks down. There are also gates that change qubit phase. The 'Hadamard' gate that takes as input a qubit that is definitely a 1 or 0 , and turns it into an indeterminate superposition. Combine a Hadamard gate with a quantum XOR gate and you have 'entangled' qubits. Entanglement, vital to QC algorithms and probably the most famous element of QC is once again simple to see in the maths, but makes little sense if explained otherwise.

Quantum computing, both adiabatic and universal, is proving a fruitful research topic. What is lagging is true public engagement. People, and most programmers, don't know degree-level maths. So, let's find new approaches to explain, and perhaps one day utilise, the power of quantum computing in more comprehensible ways.

Information on Alexis Kirke's work and further projects can be found at: www.alexiskirke.com 